\title{
Editorial
}

\section{Variational Inequalities and Vector Optimization}

\author{
Jian-Wen Peng, ${ }^{1}$ Nan-Jing Huang, ${ }^{2}$ Xue-Xiang Huang, ${ }^{3}$ and Jen-Chih Yao ${ }^{4}$ \\ ${ }^{1}$ School of Mathematics, Chongqing Normal University, Chongqing 400047, China \\ ${ }^{2}$ Department of Mathematics, University of Sichuan, Chendu, Sichuan 610064, China \\ ${ }^{3}$ School of Economics and Business Administration, Chongqing University, Chongqing 400030, China \\ ${ }^{4}$ Center for General Education, Kaohsiung Medical University, Kaohsiung 80708, Taiwan
}

Correspondence should be addressed to Jian-Wen Peng; jwpeng6@yahoo.com.cn

Received 7 February 2013; Accepted 7 February 2013

Copyright (c) 2013 Jian-Wen Peng et al. This is an open access article distributed under the Creative Commons Attribution License, which permits unrestricted use, distribution, and reproduction in any medium, provided the original work is properly cited.

As very powerful and important tools in the study of nonlinear sciences, variational inequalities and vector optimization have attracted so much attention. Over the last decades, variational inequality and vector optimization techniques have been applied extensively in such diverse fields as biology, chemistry, economics, engineering, game theory, management science, physics, and so on. The thorough study of both theory and methods about variational inequalities and vector optimization contained in the literature will help us to find new variational inequalities and vector optimization techniques for solving the practical problems.

The aim of this special issue is to present new approaches and theories for variational inequalities and vector optimization problems arising in mathematics and applied sciences.

This special issue includes 24 high-quality peer-reviewed papers that deal with different aspects of variational inequalities and vector optimization problems. These papers contain some new, novel, and innovative techniques and ideas. We hope that all the papers published in this special issue can motivate and foster further scientific works and development of the research in the area of the theory, algorithms, and applications of variational inequalities and vector optimization problems.

\section{Acknowledgments}

As guest editors for this special issue, we wish to thank all those who submitted papers for publication and many specialists who served as the reviewers. We highly appreciate the support from the editorial members of the journal, as well as the editorial staff of Hindawi Publishing Corporation. J.-W. Peng was supported by the National Nature Science Foundation of China (no. 11171363), the Special Fund of Chongqing Key Laboratory (CSTC 2011KLORSE01), and the project of the third batch support program for excellent talents of Chongqing City High Colleges.

Jian-Wen Peng Nan-Jing Huang Xue-Xiang Huang Jen-Chih Yao 


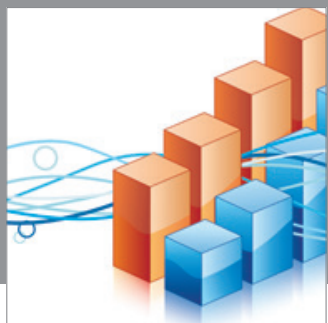

Advances in

Operations Research

mansans

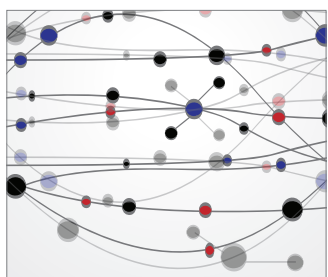

The Scientific World Journal
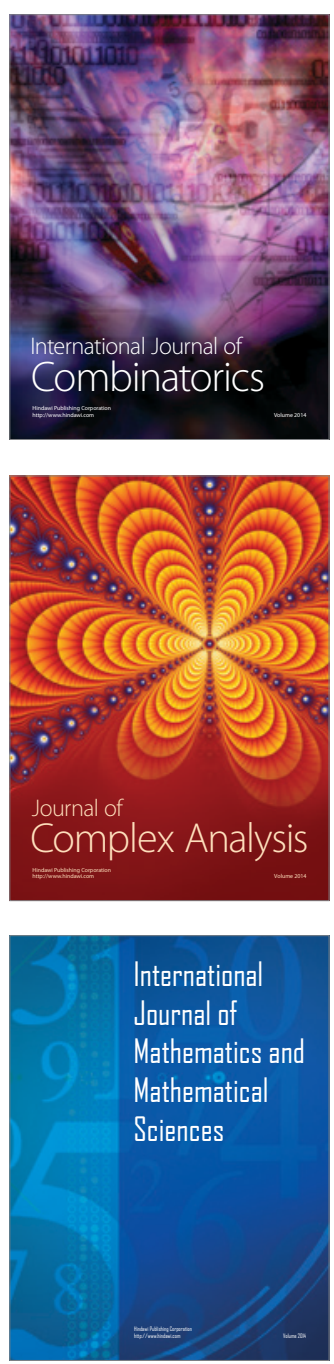
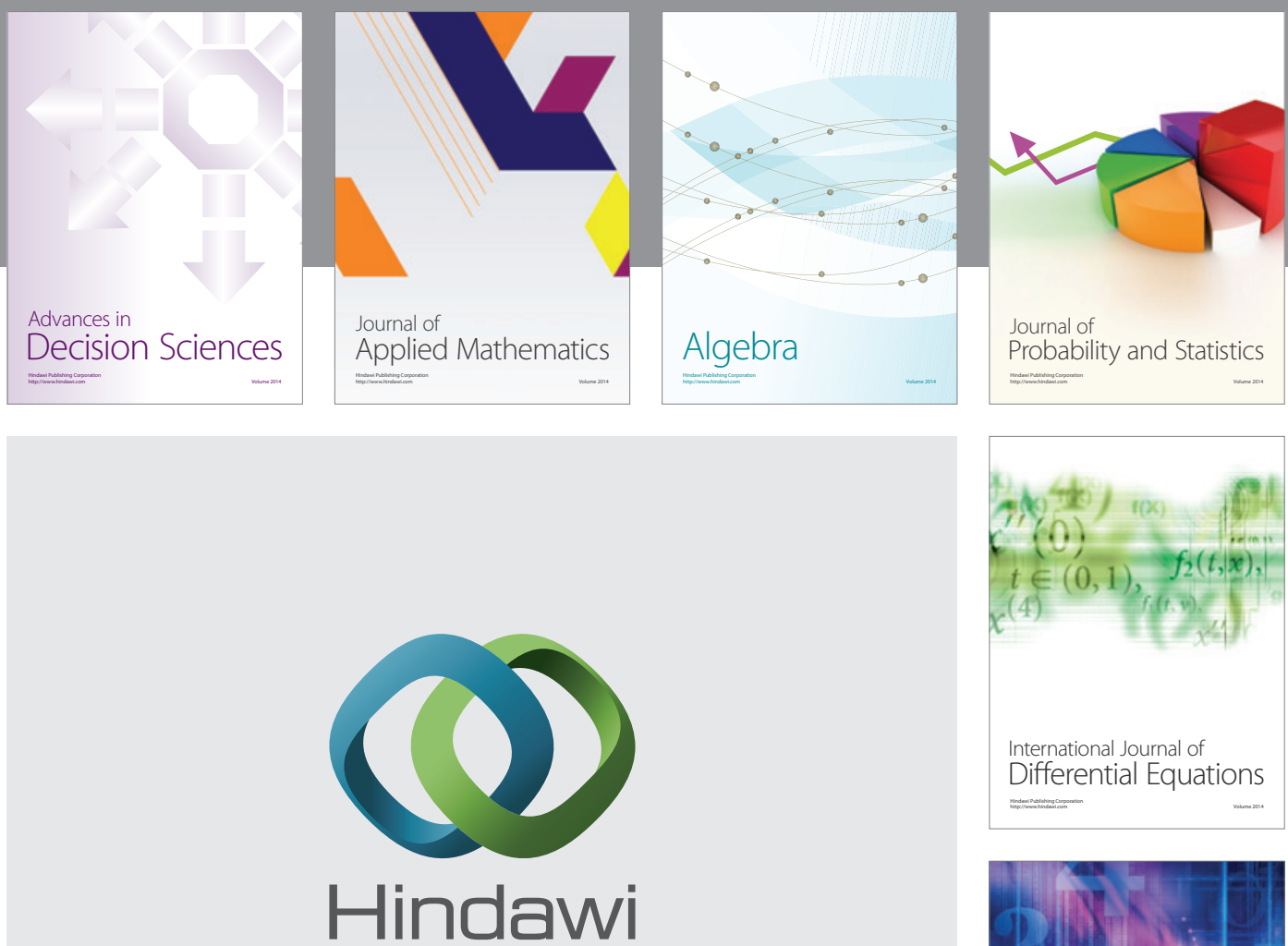

Submit your manuscripts at http://www.hindawi.com
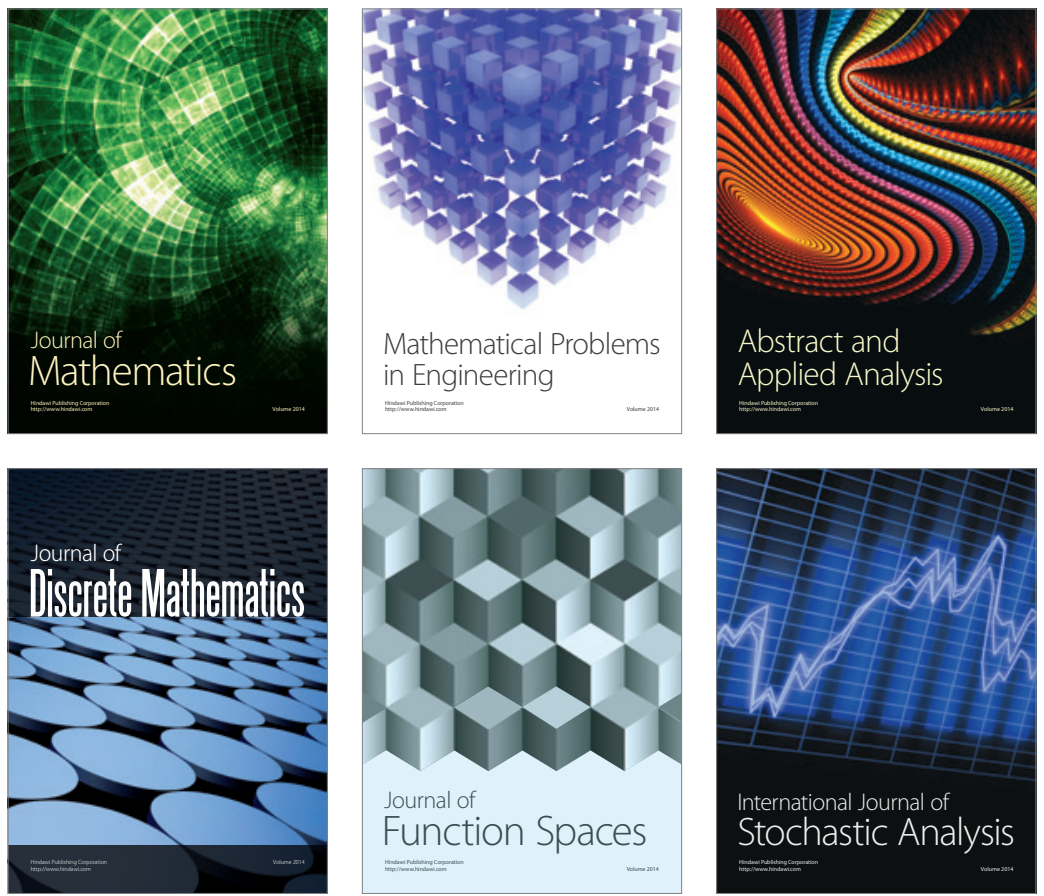

Journal of

Function Spaces

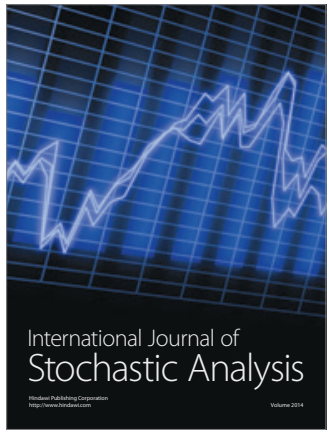

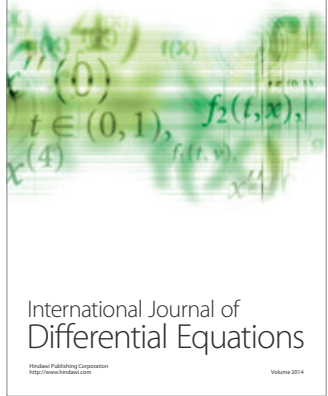
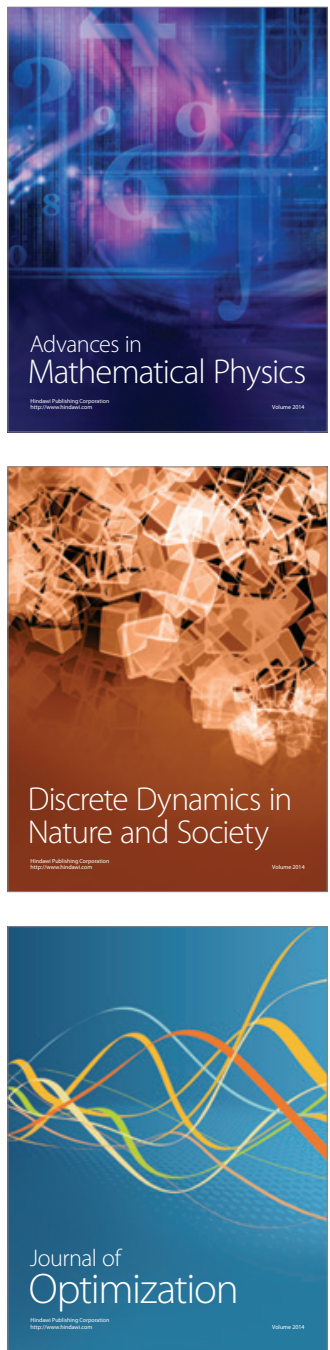